\title{
MODIFIED VACUUM-ASSISTED CLOSURE
}

\author{
Sia SF and Fong EP \\ Division of Plastic Surgery, Department of Surgery, Faculty of Medicine, University of Malaya, 50603 Kuala Lumpur, Malaysia
}

\begin{abstract}
The treatment of large ulcers is a problem. Closure with flaps and even skin grafts in compromised patients is often fraught with risks. Dressing these large wounds is also a problem, as they are often heavily exudative. We report our experience using the vacuum-assisted closure technique in managing difficult ulcers in four patients. A modification of the technique using cheap, easily available materials is described. The vacuum-assisted suction is an effective treatment for large ulcers in compromised patients. It facilitates considerable contraction of the wound, and in smaller wounds; the vacuum-assisted closure allows sufficient contracture to enable delayed primary closure. We recommend the use of this method of wound dressing and closure in large wounds particularly, in high-risk patients where extensive flap coverage or even split skin graft would incur considerable risk. (JUMMEC 2006; 9(2): 24-27)
\end{abstract}

KEYWORDS: Modified vacuum-assisted closure, difficult ulcers

\section{Introduction}

The vacuum-assisted closure (VAC) system, which uses sub-atmospheric pressure and is non-invasive, has been widely used in the management of ulcers.

In this technique, the negative pressure is applied uniformly to the wound. This allows the surrounding arterioles to dilate, increasing the local circulation. Negative pressure also removes excess fluid from the wound and reduces oedema. In addition, it also enhances wound healing by promoting the growth of granulation tissue $(I, 2)$. In many instances, it can be replaced by the use of simple, cheap, easily available material. Modification of this technique using the conventional closed disposable vacuum suction in the ward has produced equally good results.

We report our experience using a modified vacuumassisted closure technique in the management of difficult ulcers.

\section{Case Reports}

\section{Case I}

A 60 year old lady presented to us with a large, sloughy diabetic ulcer over the left inner thigh area, which had existed for a month. Following debridement, the resulting cavity measured $160 \times 120 \mathrm{~mm}$ in diameter (Figure I). Following irrigation of the wound, a I4F-size nasogastric tube was placed in the depths of the wound. The proximal end was then bought out at the edge of the cavity. The cavity was loosely packed

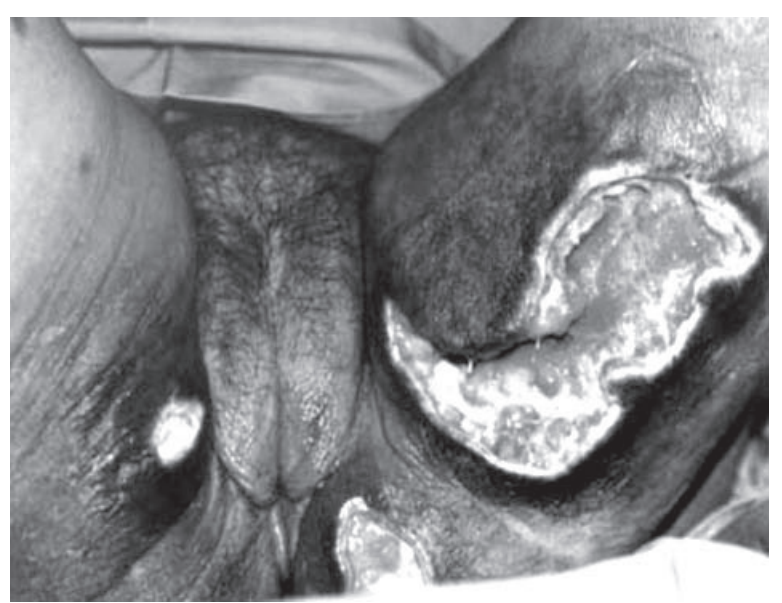

Figure I. Diabetic ulcer over the left thigh following debridement

Correspondence:

Dr Sia SF

Department of Surgery

Faculty of Medicine

University of Malaya

50603 Kuala Lumpur, Malaysia

Tel: 603-7949 3852

Email:sfsia02@um.edu.my 


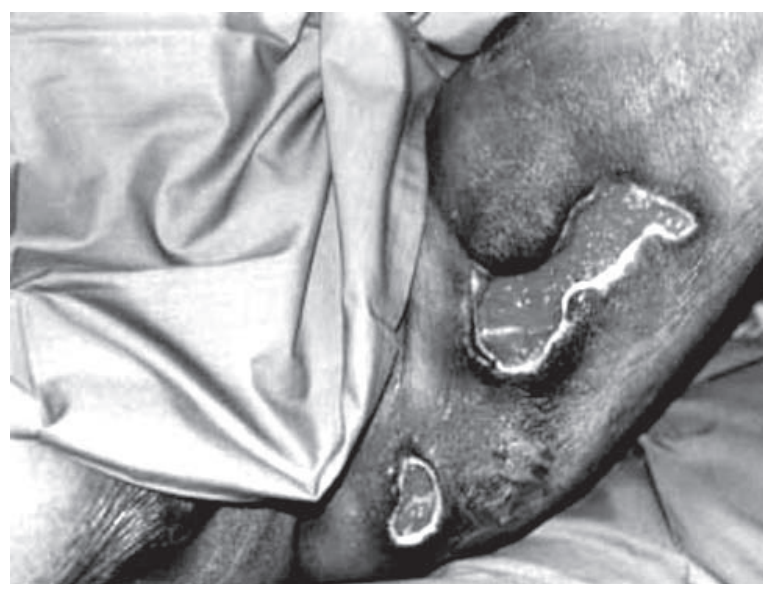

Figure 2. Diabetic ulcer over the left thigh following application of vacuum-assisted closure dressing

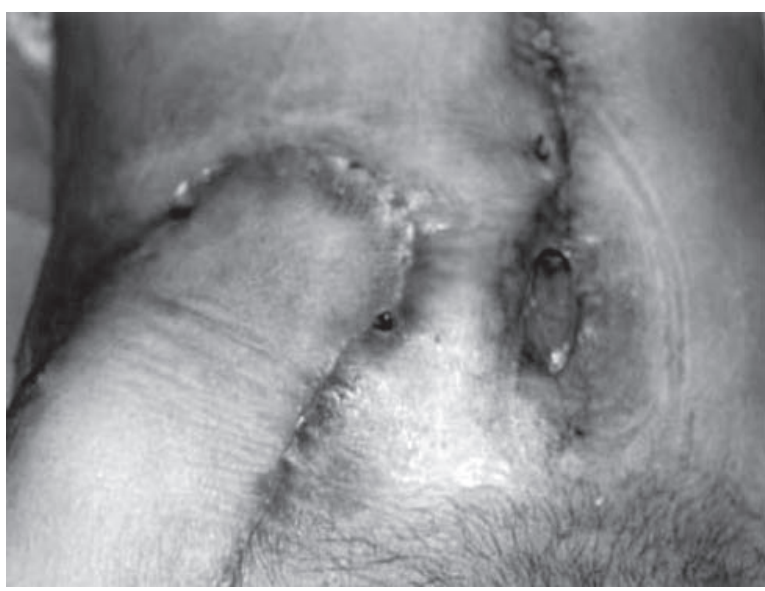

Figure 3. Dehiscence of the midline laparotomy wound

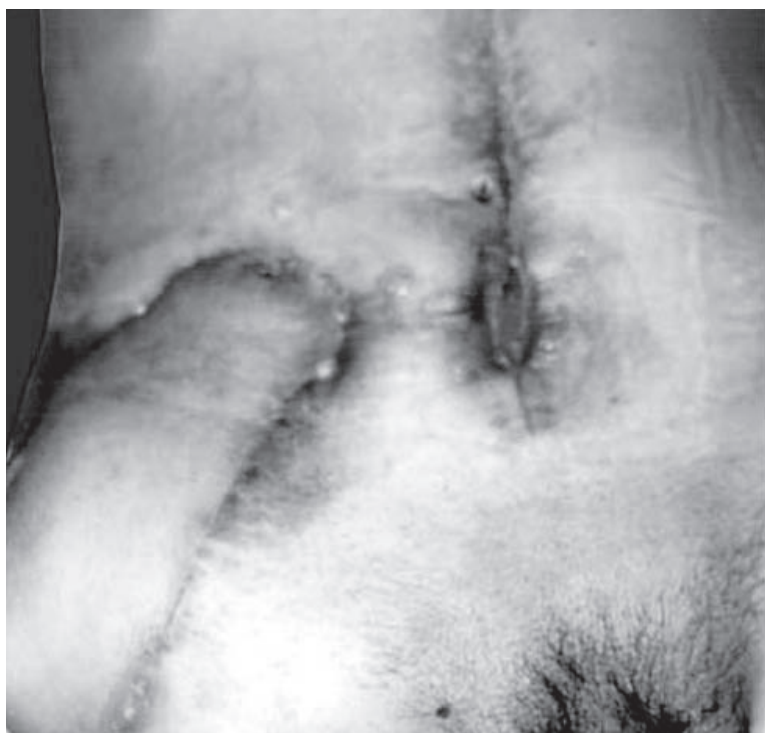

Figure 4. Wound almost closed following vacuum-assisted suction dressing with iodine-soaked gauzes. An occlusive dressing (Opsite, Smith \& Nephew, Hull, UK) was placed over the defect to achieve airtight closure, with the edges of the dressing extending $3-5 \mathrm{~cm}$ over the adjacent intact skin, converting the open wound into a controlled closed wound. The drainage catheter was connected to a wall suction vacuum unit continuously to maintain a tightly collapsed state and crinkled appearance of the occlusive dressing. The vacuum pressure was maintained at $150 \mathrm{mmHg}(-200 \mathrm{mBar})$. The wound was inspected and the dressing changed daily. Analgesia in the form of pethidine injection was given prior to each dressing change. The management of the patient's wound in this manner did not hinder her mobility, as she was instructed to disconnect the tubing from the wall unit if she wished to mobilize or use the toilet or shower. The tubing was simply plugged with a stopper during mobilization.

Within one week of treatment, the wound had contracted to one third of its original size, allowing split skin graft (Figure 2).

\section{Case 2}

A 61 year old man underwent elective right hemicolectomy for carcinoma of the caecum one year ago. He was admitted for a recurrent tumour at the right iliac region of the abdominal wall as well as the underlying small bowel. Excision of the recurrent tumour was done and the resultant abdominal wall defect closed using a loco-regional tensor fascia lata flap with underlying prolene mesh. Two days post-operatively, however, an area of wound dehiscence was noted at the midline laparotomy incision as well as the edge of the tensor fascia lata flap (Figure 3). Both wounds were connected with extensive undermining.

The wound was dressed with vacuum-assisted suction with total closure of the deep wound and the reepithelization of the surface within three weeks (Figure 4).

\section{Case 3}

A 70 year old lady had bilateral total knee replacement done for severe osteoarthritis. There was left knee surgical wound dehiscence associated with significant undermining, making the cavity double the size of the skin defect (Figure 5). Modified vacuum-assisted suction dressing was applied enabling wound contracture and split skin graft was done one week later (Figure 6).

\section{Case 4}

A 9l year old diabetic lady developed nercotizing fasciitis in the ward following a coronary artery bypass graft procedure. She had suffered a myocardial infarct 


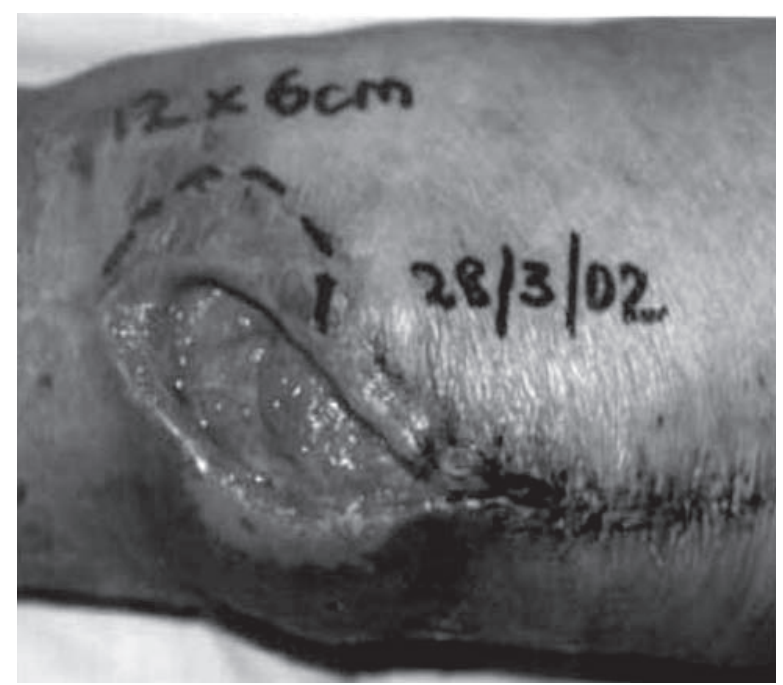

Figure 5. Wound dehiscence following total knee replacement

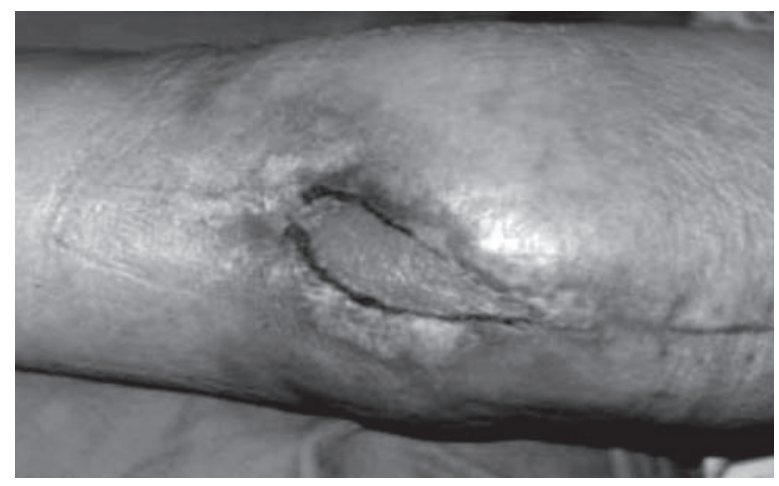

Figure 6. The wound following application of split skin graft

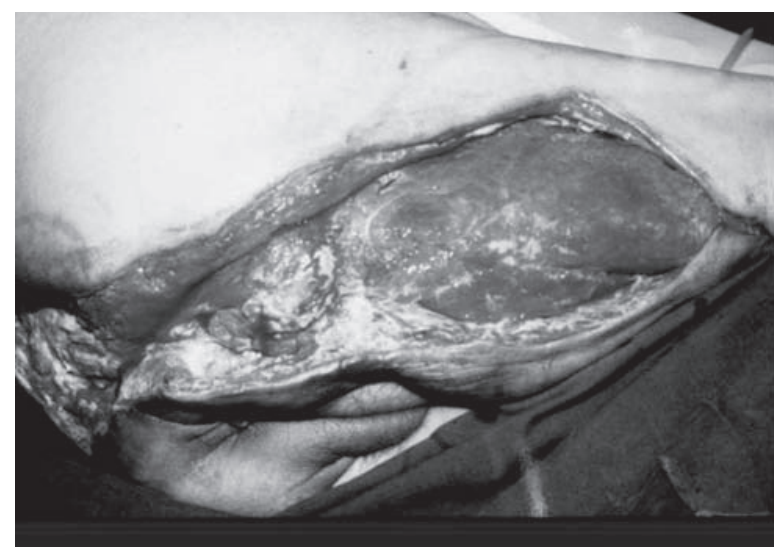

Figure 7. Wound at posterior thigh following debridement prior to the application of suction dressing

six months previously. The extensive necrotic area on the posterior aspect of the right thigh and gluteal region was debrided twice and daily dressing of the wound with modified vacuum-assisted suction was done (Figures 7 and 8).

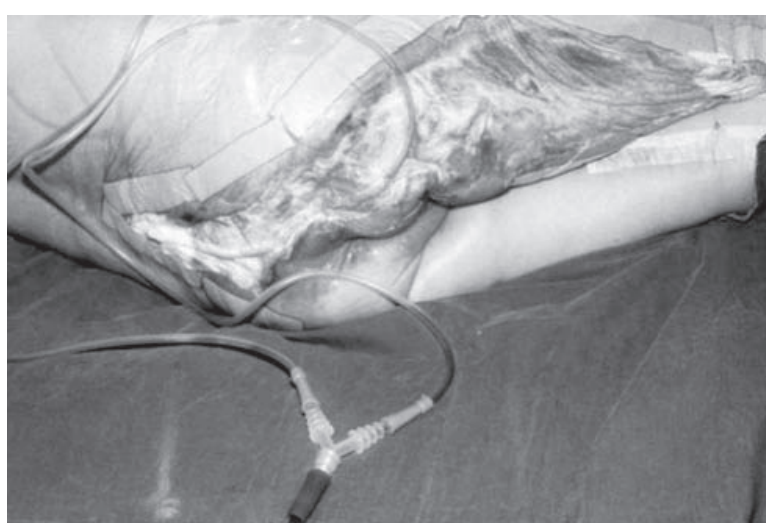

Figure 8. Vacuum-assisted suction was done with two nasogastric tubes placed in the wound bed.

Both tubes were joined with a Y-connector before connecting to the main suction tubing.

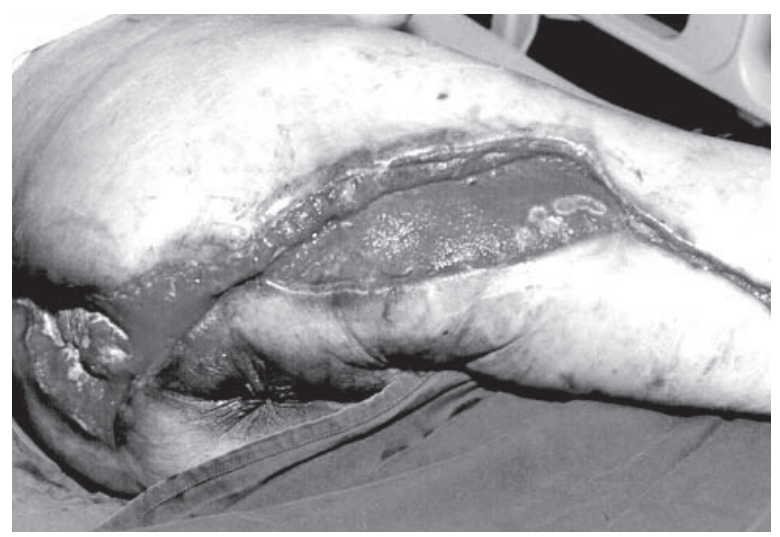

Figure 9. The wound at one month just prior to split skin graft

After one month, the wound had contracted considerably with a healthy granulating bed allowing a simple split skin graft procedure (Figure 9).

\section{Discussion}

In 1911 the application of mechanical stress onto wounds was described (3), which accelerated the healing process by promoting angiogenesis and tissue growth. Mechanical deformation of the cells resulted in an increase in protein and matrix molecule synthesis.

The use of topical negative pressure onto wounds has been well published and its value as a treatment modality well documented. Application of controlled sub-atmospheric pressure to a wound remove the excess interstitial fluid, reduces tissue turgor, decreases the oedema and enhances local blood flow. This in turn will improve the local tissue oxygenation and nutrient supply, increase the outflow of toxin and 
inhibitory factors and decrease tissue bacterial levels $(I, 2,4,5)$. Thus, healthy granulating tissue appears much more rapidly. The original conventional sub-atmospheric pressure system which employs a medical grade reticulated polyethane ether foam dressing with embedded non-collapsible drainage tube is costly and not easily available especially in small district hospitals. Modifying the technique by using absorbent gauze, occlusive dressing, nasogastric tubing and continuous controlled wall suction or a portable vacuum suction pressure machine, we have found the technique simple and effective both in terms of cost and result. It is easily taught to nursing staff. There is immediate improved local hygiene with rapid formation of granulation tissue.

Some series have reported that exposed bone and tendon in orthopaedic patients may be covered with VAC technique (6). In our patient (Case 3), the skin and soft tissue cover over the knee was thin with significant oedema making retraction of tissue off the underlying bone and bony exposure a real risk. Modified vacuum-assisted dressing provided rapid wound contracture.

Although in our series, the negative pressure is connected to wall suction apparatus, the therapy did not require undue immobilization of any of these patients. The patients were allowed to disconnect the drainage tube from the wall suction entry and ambulate as advised.

Bacterial counts were reported to drop from approximately $10^{7}$ per gram of tissue to $10^{2}$ to $10^{3}$ per gram tissue in animal studies and in human samples by day $5(1,2)$. Bacterial levels of less than $10^{5}$ per gram of tissue are associated with progressive successful wound healing.

The four patients reported here were staying in the ward throughout the treatment. No difficulties were encountered during the dressing procedure. Successful healing without surgical intervention was obtained in Case 2. In the other three patients, a simple split skin graft procedure could be performed on the healthy granulating contracted wound.

Some patients may require analgesia with dressing change. Some degree of pain may be experienced when the tubing is first connected to the suction apparatus and sub-atmospheric pressure is transmitted to wound. Gradual negative pressure increment resulted in better tolerance as opposed to a sudden increment. Occasionally, movement caused displacement of the occlusive dressing leading to loss of vacuum. To remedy this situation, the patients were advised to check the seal (i.e., constant airtight closure with crinkling of the occlusive dressing). Should an air leak be present, this could simply be resolved by reinforcing with additional layer of occlusive dressing, overlapping the edge of the first layer. However, if excess egress of exudate or soiling of the wound with faecal material accompanies loss of seal, the dressing should be changed completely.

Bleeding from the wound is another possible complication of using the vacuum-assisted suction. This may occur during dressing change. Hemostasis can be easily secured with pressure.

\section{Conclusion}

The modified vacuum-assisted suction technique is an effective treatment for large or difficult ulcers in compromised patients. It enhances and facilitates considerable contraction of the wound and in smaller wounds; the vacuum-assisted suction allows sufficient contracture to enable delayed primary closure. We recommend this method of wound dressing and closure in large wounds particularly, in high risk and compromised patients. Cost efficiency can be optimized in the care of many patients.

\section{References}

I. Argenta LC, Morykwas MJ. Vacuum-assisted closure: a new method for wound control and treatment. Clinical experience. Ann Plast Surg 1997; 38(6): 563-567.

2. Morykwas MJ, Argenta LJ, Shelton-Brown El, et al. Vacuum-assisted closure: a new method for wound control and treatment. Animal studies and basic foundation. Ann Plast Surg 1997; 38(6): 553-562.

3. Thoma R. Ueber die Histomechanik des Gesfasssystems und die Pathogenase der Angioskleroose. Virchows Arch. F. Pathol. Anat 1911; 204:1.

4. ShaerWD. Inexpensive vacuum-assisted closure employing a conventional disposable closed suction drainage system. Plast Reconstr Surg 200 I ( ); 107: 292-293.

5. Collier M. Know how: vacuum-assisted closure (VAC). Nurs Times 1997; 93: 32-33.

6. DeFranco AJ, Argenta LC, Marks MW. The use of vacuum-assisted closure theraphy for the treatment of lower extremity wound with exposed bone. Plast Reconstr Surg 200I; 108(5): I |84- | | | |. 